\title{
Novel Methylcobalamin Nasal Spray versus Intramuscular Injection: A Randomized, Crossover Comparative Bioavailability Study
}

\section{Dave $\mathbf{H}^{1}$, Agarwal $\mathbf{V}^{2}$, Maseeh $\mathrm{A}^{2 *}$, and Patel $\mathrm{KR}^{2}$}

${ }^{1}$ Head, Clinical Service, Raptim Research Limited, A-242, A-226, TTC Industrial Area, Near Mahape Depot, Mahape MIDC, Navi Mumbai-Mumbai, Maharashtra, India

${ }^{2}$ Medical Services Department, Troikaa Pharmaceuticals Limited, Ahmedabad, India

*Corresponding author: Dr. Arun Maseeh, MD, Internal Medicine, Senior Vice President-Medical, Troikaa Pharmaceuticals Limited, Commerce House-1, Satya Marg, Bodakdev, Ahmedabad, India, 380054, Tel: +917926856242; E-mail: arunmaseeh@troikaapharma.com

Received: 15 Dec, 2020 | Accepted: 11 Jan, 2021 | Published: 19 Jan, 2021

Citation: Dave H, Agarwal V, Maseeh A, Patel KR (2021) Novel Methylcobalamin Nasal Spray versus Intramuscular Injection: A Randomized, Crossover Comparative Bioavailability Study. Int J Endocrinol Metab Disord 7(1): dx.doi.org/10.16966/2380-548X.171

Copyright: (c) 2021 Dave $\mathrm{H}$, et al. This is an open-access article distributed under the terms of the Creative Commons Attribution License, which permits unrestricted use, distribution, and reproduction in any medium, provided the original author and source are credited.

\section{Abstract}

Background/Objective: Methylcobalamin Nasal Spray (NasoB12 ${ }^{\circledR}$, Troikaa, India) has been recently developed to overcome the drawbacks of currently available therapeutic routes. This study was designed to compare pharmacokinetics, safety and tolerability of methylcobalamin nasal spray with intramuscular injection.

Subjects/Methods: This was a randomized, single dose, open label, two way crossover comparative bioavailability study on 18 healthy volunteers. Participants were administered either $500 \mathrm{mcg}$ of methylcobalamin via nasal spray $(250 \mathrm{mcg} / 0.05 \mathrm{ml}$, one actuation in each nostril) or $100 \mathrm{mcg} / 0.2$ $\mathrm{ml}$ of methylcobalamin via intramuscular injection. Serial blood samples were collected in each study period, following drug administration and plasma concentrations of $B 12$ were measured. Time to observe maximum drug concentration $\left(T_{\max }\right)$, maximum plasma concentration $\left(C_{\max }\right)$ and area under the concentration-time curve (AUC) were compared.

Results: Vitamin B12 absorption data from nasal spray as compared to intramuscular Injection showed mean [ $T_{\max }$ ] of 0.2 vs. 1.15 hours for injection. When compared without dose normalization, the nasal spray demonstrated similar bioavailability to the Intramuscular Injection (ratio of $104.77 \%$ for $\mathrm{AUC}_{0 \text {-t }}$ and $101.85 \%$ for $A \cup C_{0 \text {-inf }}$. Upon dose normalization the comparative bioavailability of intranasal spray versus intramuscular injection was observed to be $20 \%$. Higher $C_{\text {max }}$ for intranasal spray was observed as compared to intramuscular injection without dose normalization (197.49\%); however, upon dose normalization $\mathrm{C}_{\max }$ was $39.50 \%$. The safety evaluations indicated that both the treatments were well tolerated.

Conclusions: Vitamin B12 is adequately absorbed after intranasal application of methylcobalamin nasal spray and can be used as a safe and effective alternative to current options.

\section{Introduction}

Vitamin B12 (B12) also known as cobalamin is a cofactor for major reactions in the body which are critical for DNA synthesis, red blood cell formation and for neurologic function [1]. B12 deficiency may be caused by factors such as improper dietary intake, problems with bioavailability or malabsorption $[2,3]$.

B12 deficiency is traditionally treated with oral formulations, or, preferably, repeated intramuscular injections of cobalamin. The intramuscular injections are painful, inconvenient and often associated with adverse reactions (e.g. abscess formation, erythema, and hematoma) $[4,5]$. Furthermore, administration of injectables requires health care professionals, which increases the economic burden on the healthcare system. This may impact patient compliance and result in discontinuation of the treatment [6,7]. Daily oral supplementation of Vitamin B12, dependent on Intrinsic Factor (IF),
[8] is hindered by increasing age, long-term concomitant metformin or antacid therapy, gastric surgery and in patients with Pernicious Anemia $[1,9,10]$.

The nasal route of drug administration is attractive due to its easy accessibility, the large surface area, high vascularization, porous endothelial basement membrane, potential to bypass first-pass metabolism, and possible higher systemic bioavailability versus the oral route $[11,12]$. An Intranasal formulation overcomes the drawbacks of intramuscular formulation: it is painless, self-administrable, has improved compliance, eliminates the need for administration by health professionals, prevents injection site complications and reduces the overall cost of therapy [13].

The Nasal route has been studied for delivery of B12 in various populations $[6,14,15]$. Cyanocobalamin Nasal Spray has received regulatory approval. However, based on the pharmacokinetic data in 
the label of cyanocobalamin nasal spray, the drug is not used for the treatment of B12 Deficiency in patients with pernicious anemia, but rather, used for maintenance therapy, one month after the treatment and remission following $100 \mathrm{mcg}$ intramuscular Vitamin B12 therapy and also used as a supplement in other deficiencies [16].

Cyanocobalamin has to be enzymatically converted into one of two metabolically active coenzyme forms, (i.e. methylcobalamin and 5-deoxyadenosylcobalamin) for its effects in the body. Nutritional deficiencies and defects in enzymes reduce the body's ability to synthesize active forms [17]. Since, the active form methylcobalamin bypasses several enzymatic reactions required for the formation of functional forms, better retained in the body as compared to Cyanocobalamin [17], it is prudent to consider methylcobalamin in treatment of Vitamin B12 deficiency in place of Cyanocobalamin.

Looking at the formulation advantages offered by intranasal form over other currently available formulations, a novel Intranasal Methylcobalamin Spray $(500 \mathrm{mcg} / 0.1 \mathrm{~mL}$ ) has been developed (Troikaa Pharmaceuticals Limited). To the best of our knowledge, the current Phase I study represents the first in human study to assess the safety and pharmacokinetics of the novel Intranasal Methylcobalamin Sprayin comparison to currently marketed Intramuscular Methylcobalamin Injection. This study was conducted as a randomized, single dose, open label, crossover study in healthy human subjects.

\section{Methods}

Clinical part of the study was carried out at Raptim Research Ltd. The study was approved by the Etiikka (Independent Ethics Committee) IEC and was conducted in compliance with the requirements of ICH (International Conference on Harmonization) E6 'Guideline for Good Clinical Practice' and World Medical Association's Declaration of Helsinki.

\section{Selection of Subjects}

Eighteen (18) healthy, adult, male human subjects, aged between 18 to 45 years \& body mass index between 18.5 to $24.9 \mathrm{~kg} / \mathrm{m}^{2}$ participated in the study. All the subjects provided written informed consent to participate in the study prior to enrolment and were free to withdraw at any time during the study. Subjects were only included if their serum total B12 level was in the range of 200 to $900 \mathrm{pg} / \mathrm{ml}$ at baseline laboratory evaluation and had other normal laboratory values as determined by hematological tests, biochemistry, urine analysis and ECG in correlation with clinical findings. Subjects with nasal polyp, running nose, nasal congestion, allergic rhinitis, respiratory tract infection and with known hypersensitivity to methylcobalamin were excluded. All the subjects complied with inclusion and exclusion criteria set out in the study protocol.

\section{Study design and drug administration}

This was a 1:1 randomized, single dose, open label, two-way crossover comparative bioavailability study. A sample size of 18 subjects was considered adequate to determine the significant formulation difference between the test product and reference product. A wash out period of 15 days was kept between the two treatments. The study was conducted from 8 to 29 January, 2014. Subjects were housed from approximately 12.00 hours prior to drug administration. As per the randomization schedule (generated by SAS ${ }^{\circledR}$ software (SAS Institute Inc., USA) (version 9.2), each subject received either $0.1 \mathrm{~mL}$ $(500 \mathrm{mcg})$ test nasal spray $(250 \mathrm{mcg} / 0.05 \mathrm{ml}$ in each nostril) or $0.2 \mathrm{~mL}$ $(100 \mathrm{mcg})$ reference IM injection of methylcobalamin (Methycobal manufactured by Eisai, Japan) after overnight fasting for at least 10.00 hours in each period.

\section{Safety}

All subjects were assessed for safety and tolerability during the study period. This included vital signs, well-being, local tolerability, ECG, Biochemistry, Hematology and Urinalysis.

Blood samples for analysis of B12: Blood samples were collected at -10.00 hours, -6.00 hours, 0.00 hours (pre-dose samples for baseline) and at $0.16,0.25,0.33,0.50,0.75,1.00,1.25,1.50,1.75,2.00,2.50$, $3.00,4.00,6.00,8.00,12.00,24.00,48.00,72.00,96.00$ and 120.00 hours post-dose in pre-labeled EDTA vacutainers. Blood samples were centrifuged at $4000 \mathrm{rpm}$, for 10 minutes at $8^{\circ}-10^{\circ} \mathrm{C}$ for plasma separation. After centrifugation the plasma samples were separated and transferred into respective pre-labeled amber colored eppendorf tubes into two aliquots.

Vitamin B12 estimation in plasma: The plasma samples were assayed for Total Vitamin B12 concentration using a validated Electrochemiluminescence method using Roche Cobas e411 instrument at APL Institute of Clinical Laboratory \& Research Pvt. Ltd. A validated Electro-chemiluminescent Immunoassay kit from Roche Diagnostics having a calibration range of $30-2,000 \mathrm{pg} / \mathrm{ml}$ was used for analysis. Further validation of this kit was done for this study to extend its analytical range up to $15,000 \mathrm{pg} / \mathrm{ml}$. The concentration below the Limit of quantification (BLQ) was considered as 'zero' for all pharmacokinetic and statistical evaluation. Analyst did not have access to the randomization schedule till completion of the analysis.

Pharmacokinetic and statistical analysis: The primary pharmacokinetic parameters $\mathrm{C}_{\max }, \mathrm{AUC}_{0-\mathrm{t}}, \mathrm{AUC}_{0 \text {-inf }}$ and secondary $\mathrm{PK}$ parameters $t_{1 / 2}, K_{e l}$ and $T_{\max }$ were calculated by the non-compartmental approach using WinNonlin professional (version 5.3). B12 is an endogenous compound and therefore pharmacokinetic analysis was done both with and without baseline correction. The baseline correction was done by subtracting the average pre-dose levels from subsequent post-dose levels. This was desirable to evaluate the contribution of B-12 from diet and endogenous perturbations.

The two treatments in this study had five fold differences in total dose and were given by different routes. Hence, dose normalization was also done for pharmacokinetic parameters such as $\mathrm{C}_{\max }, \mathrm{AUC}_{0-\mathrm{t}}$ and $\mathrm{AUC}_{0 \text {-inf }}$ to predict the relative bioavailability at the same dose.

The statistical analysis was performed using SAS system (SAS institute USA, version 9.2) for both dose-normalized and nonnormalized data after baseline correction. Two one-sided ANOVA was performed on log transformed pharmacokinetic parameters $\mathrm{C}_{\text {max }}, \mathrm{AUC}_{0-\mathrm{t}}$ and $\mathrm{AUC}_{0 \text {-inf }}$ (at $\alpha=0.05$ ), to determine the difference between the test and reference products. Non-Parametric Analysis of untransformed $\mathrm{T}_{\text {max }}$ using Wilcoxon Signed Rank Test.

\section{Results}

\section{Subject demographics and disposition}

Eighteen [18] subjects who met the selection criteria participated in the study. Total 15 subjects completed both the periods of the study. Two subjects did not report to the facility for Period II, hence dropped out from the study and one subject withdrew due to personal reason before dosing in period II. Thus, data of the 15 subjects who completed the study was considered for analysis. Baseline demographic data of the study populations are given in table 1 and subject disposition is described in figure 1.

\section{Safety and tolerability}

Both the treatments (Nasal Spray and IM injection) were well tolerated. No serious adverse events were reported during this study. 
Table 1: Subjects demographic characteristics at baseline $(\mathrm{N}=18)$.

\begin{tabular}{|c|c|c|c|c|c|c|}
\hline & Age (Years) & Weight (kg) & Height (cm) & BMI $\left(\mathrm{kg} / \mathrm{m}^{2}\right)$ & Gender & Race \\
\hline Mean \pm SD & $29.8 \pm 5.8$ & $64.2 \pm 7.3$ & $170.0 \pm 5.1$ & $22.2 \pm 2.0$ & \multirow{2}{*}{ Male } & \multirow{2}{*}{ Asian } \\
\hline Median & 29.0 & 63.0 & 170.0 & 22.7 & & \\
\hline
\end{tabular}

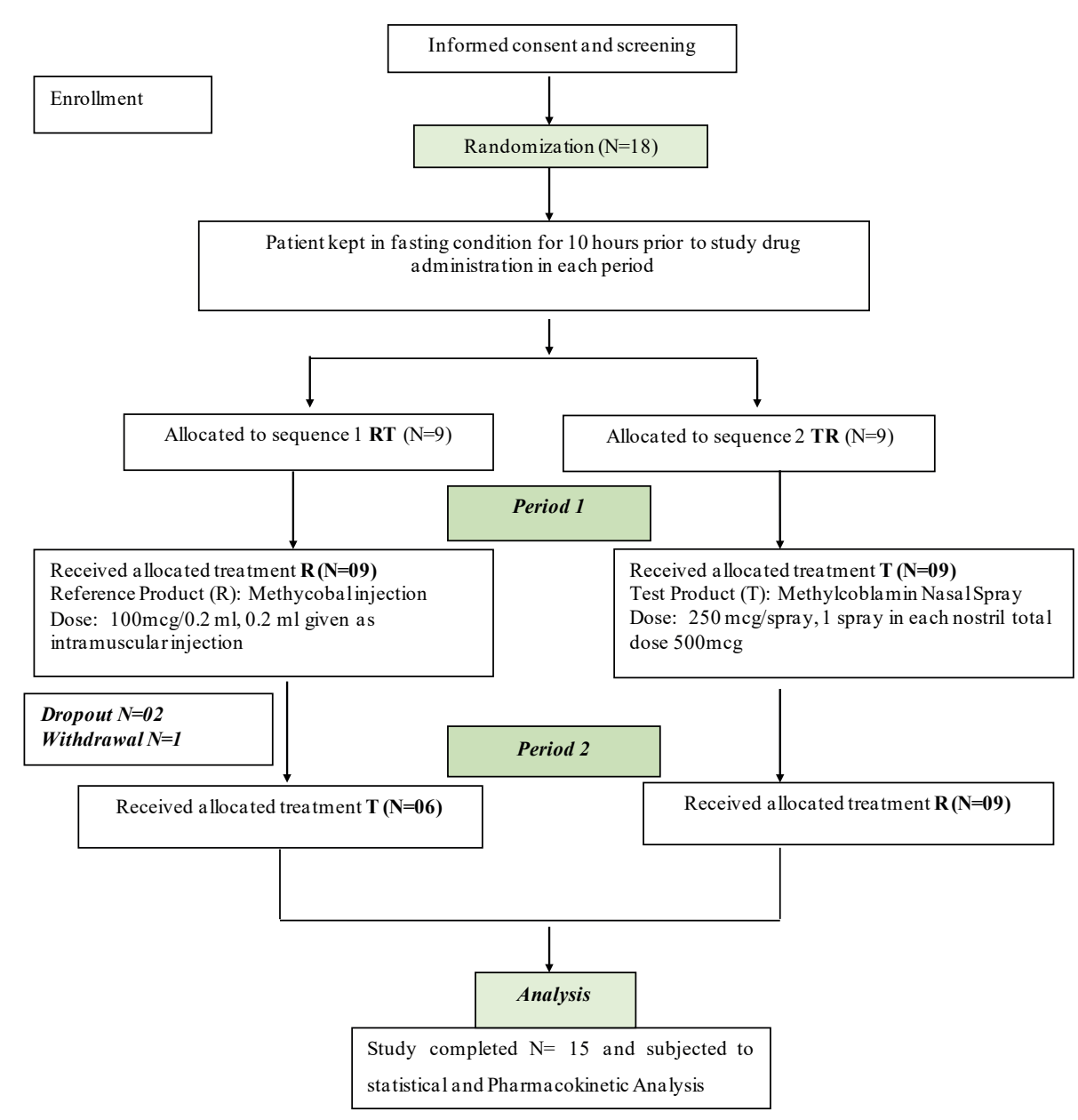

Figure 1: Study flow chart.

One adverse event (dizziness) was reported in period I of the study in a subject after administration of nasal spray. It was resolved on the same day within 10 minutes of onset. The adverse event was mild, and judged as probably related to study drug administered, the subject was monitored until resolution of the adverse event.

The post-study clinical laboratory value of two subjects showed an elevated level of bilirubin direct in comparison to pre-study values; these adverse events were considered as mild and judged as possibly related to the study drug administered.

One subject had decreased level of serum B12 post-study in comparison to pre-study values. This adverse event was mild and judged as not related to the study drug administered. All these subjects were followed up for safety assessment. The follow up results were found to be within acceptable range.

There were no clinically significant findings in the vital signs or ECG recordings of all participated subjects in the post-study safety assessment. There was no Serious Adverse Event (SAE) reported in the study including any nasal route-specific SAE. Hence the test product and reference product were comparable in the safety profile.

\section{Pharmacokinetics}

Mean plasma B12 concentration $v s$. time profile for both treatments is shown in figure 2 .

The mean \pm SD baseline plasma B12 levels for test (Intranasal) and reference (Intramuscular) products were $396.14 \pm 98.66 \mathrm{pg} / \mathrm{ml}$ and $368.01 \pm 55.83 \mathrm{pg} / \mathrm{ml}$ respectively.

Intranasal methylcobalamin spray resulted in an earlier peak concentration with a mean $\mathrm{T}_{\max }$ of 0.2 hours as compared to 1.15 hours, after IM injection of Methylcobalamin.

The derived pharmacokinetic parameters for both treatments with and without baseline correction and with and without dose normalization are summarized in table 2. 


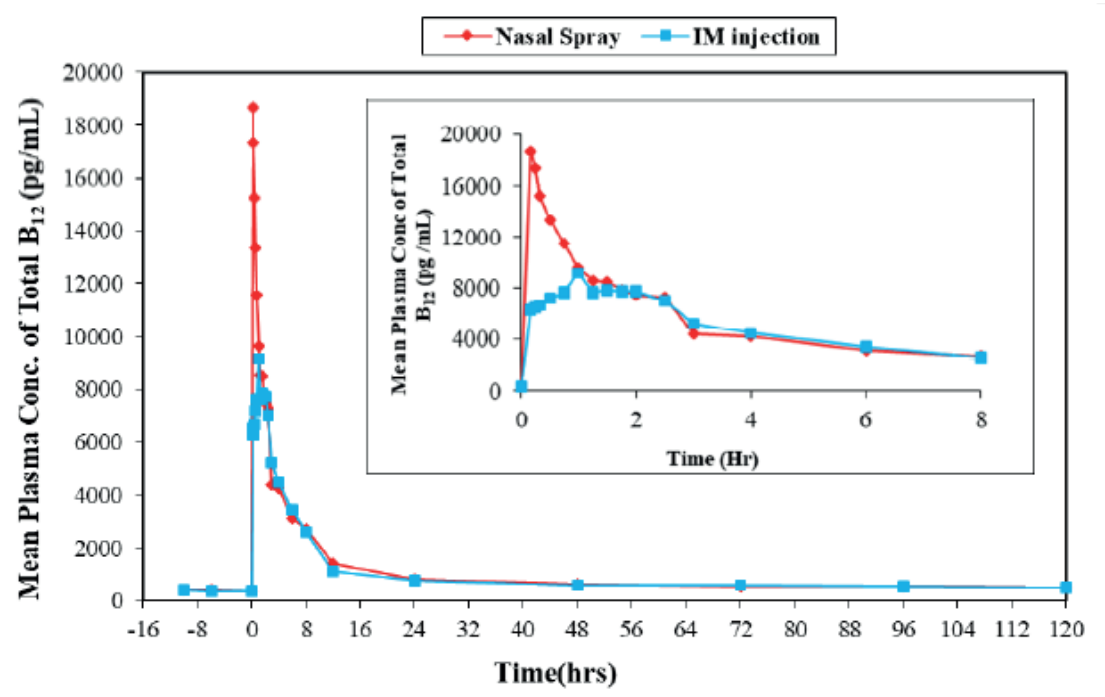

Figure 2: Mean Plasma Concentration time profile of total vitamin B12 after administration of test (nasal spray) and reference (IM injection) without baseline correction.

Inset: Mean Plasma Concentration time profile of total vitamin B12 up to 8 hours after administration of test (nasal spray) and reference (IM injection) without baseline correction.

Table 2: Comparative pharmacokinetic parameters of $500 \mu \mathrm{g}$ test (nasal spray) and $100 \mu \mathrm{g}$ reference (IM injection).

\begin{tabular}{|c|c|c|c|c|}
\hline \multirow{2}{*}{$\begin{array}{l}\text { Pharmacokinetic } \\
\text { Parameter }\end{array}$} & \multicolumn{2}{|c|}{ Without Baseline Correction } & \multicolumn{2}{|c|}{ With Baseline Correction } \\
\hline & $\begin{array}{l}\text { Test Product } \\
\text { Mean } \pm \text { SD }\end{array}$ & $\begin{array}{l}\text { Reference Product } \\
\text { Mean } \pm \text { SD }\end{array}$ & $\begin{array}{l}\text { Test Product } \\
\text { Mean } \pm \text { SD }\end{array}$ & $\begin{array}{c}\text { Reference Product } \\
\text { Mean } \pm \text { SD }\end{array}$ \\
\hline$C_{\max }(\mathrm{pg} / \mathrm{ml})$ & $19779.47 \pm 6726.28$ & $10263.53 \pm 3818.40$ & $19383.32 \pm 6766.21$ & $9895.52 \pm 3816.05$ \\
\hline$C_{\text {maxDN }}(p g / m l)$ & $39.56 \pm 13.45$ & $102.64 \pm 38.18$ & $38.77 \pm 13.53$ & $98.96 \pm 38.16$ \\
\hline$A \cup C_{0-t}\left(p g^{*} h r / m l\right)$ & $123399.31 \pm 31342.86$ & $114320.43 \pm 20921.10$ & $75533.03 \pm 29739.19$ & $70905.39 \pm 18740.95$ \\
\hline$A \cup C_{0-t D N}(p g * h r / m l)$ & $246.80 \pm 62.69$ & $1143.20 \pm 209.21$ & $151.07 \pm 59.48$ & $709.05 \pm 187.41$ \\
\hline$A \cup C_{0 \text {-inf }}\left(p g^{*} h r / m l\right)$ & $243363.97 \pm 120348.80$ & $262529.22 \pm 77288.93$ & $84243.44 \pm 29729.80$ & $86604.67 \pm 31352.09$ \\
\hline$A U C_{0 \text {-inf DN }}\left(p g^{*} \mathrm{hr} / \mathrm{ml}\right)$ & $486.73 \pm 240.70$ & $2625.29 \pm 772.89$ & $168.49 \pm 59.46$ & $866.05 \pm 313.52$ \\
\hline $\mathrm{T}_{\max }(\mathrm{hr})$ & $\begin{array}{c}0.20 \pm 0.06 \\
0.16^{\#}\end{array}$ & $\begin{array}{c}1.15 \pm 0.69 \\
1.00^{\#} \\
\end{array}$ & $\begin{array}{c}0.20 \pm 0.06 \\
0.16^{\#}\end{array}$ & $\begin{array}{c}1.15 \pm 0.69 \\
1.00^{\#} \\
\end{array}$ \\
\hline$t_{1 / 2}(h r)$ & $164.11 \pm 162.6$ & $209.13 \pm 98.57$ & $49.54 \pm 37.88$ & $65.19 \pm 37.02$ \\
\hline $\mathrm{K}_{\mathrm{el}}(\mathrm{hr}-1)$ & $0.0118 \pm 0.0111$ & $0.0048 \pm 0.0053$ & $0.0235 \pm 0.0151$ & $0.0206 \pm 0.0251$ \\
\hline
\end{tabular}

AUC: Area Under the plasma Concentration-time curve; $\mathrm{AUC}_{0 \text {-inf }}$-the area under the plasma concentration versus time curve from time zero to infinity; $\mathrm{C}_{\max }$ - maximum plasma concentration; $\mathrm{T}_{\max ,}$-time to observed maximum drug concentration in plasma $\mathrm{t}_{1 / 2}$ elimination half-life; $\mathrm{K}_{\mathrm{e}}$ - elimination rate constant; DN-dose Normalized to Methylcobalamin $1 \mathrm{mcg}$ dose. \#Median

The ratio of geometric least square means (\%), $90 \% \mathrm{CI}$ and within subject variability for both dose-normalized and non-normalized data is summarized in table 3 .

Before dose normalization, total exposure for B12 was similar for nasal spray and IM administration, with a test/reference ratio of geometric least square means of $104.77 \%$ and $101.85 \%$ for $\mathrm{AUC}_{0-\mathrm{t}}$ and $\mathrm{AUC}_{0 \text {-inf }}$ respectively. However, the mean maximum concentration of B12 in plasma after administration of nasal spray was approximately twice as compared to IM injection as represented by the test/reference ratio of geometric least square means of $197.49 \%$ for $C_{\max }$.

After dose-normalization, the bioavailability of nasal spray as compared to IM injection was $\sim 20 \%$ as represented by the test/ reference ratio of geometric least square means of $20.95 \%$ and $20.37 \%$ for $\mathrm{AUC}_{0-\mathrm{t}}$ and $\mathrm{AUC}_{0 \text { inf }}$ respectively. Also the mean maximum concentration of B12 in plasma after administration of nasal spray was approximately $\sim 40 \%$ as compared to IM injection as represented by 
Table 3: Summary of the statistical analysis $(n=15)$.The ratio of geometric least square means $(\%), 90 \% \mathrm{Cl}$ and within subject variability for both dosenormalized and non-normalized data.

\begin{tabular}{|c|c|c|c|}
\hline Pharmacokinetic parameters & $\begin{array}{l}\text { Ratio of geometric least square means } \\
\text { (\%) }\end{array}$ & $90 \% \mathrm{Cl}$ & Intrasubject CV (\%) \\
\hline \multicolumn{4}{|l|}{ Without Dose Normalization } \\
\hline $\mathrm{C}_{\max }$ & 197.49 & $160.48-243.05$ & 32.24 \\
\hline $\mathrm{AUC}_{0-\mathrm{t}}$ & 104.77 & $87.56-125.37$ & 27.70 \\
\hline $\mathrm{AUC}_{0 \text {-inf }}$ & 101.85 & $82.42-125.86$ & 32.91 \\
\hline \multicolumn{4}{|l|}{ With Dose Normalization } \\
\hline $\mathrm{C}_{\max }$ & 39.50 & $32.09-48.61$ & 32.24 \\
\hline $\mathrm{AUC}_{0-\mathrm{t}}$ & 20.95 & $17.51-25.07$ & 27.70 \\
\hline $\mathrm{AUC}_{0 \text {-inf }}$ & 20.37 & $16.48-25.17$ & 32.91 \\
\hline
\end{tabular}

the test/reference ratio of geometric least square means of $39.50 \%$ for $\mathrm{C}_{\max }$.

\section{Discussion}

This was a randomized, single dose, open label, crossover comparative bioavailability study which aimed to determine the safety and comparative bioavailability of the novel intranasal methylcobalamin spray with Methylcobalamin IM injection. Our study represents the first-in-human results of the absorption of methylcobalamin through the nasal route in healthy volunteers.

The B12 dose in this study was $500 \mathrm{mcg}$ for methylcobalamin nasal spray and $100 \mathrm{mcg}$ for IM injection, this is in line with the study carried out for Nascobal ${ }^{\circ}$ nasal spray where $500 \mathrm{mcg}$ dose was used for nasal formulations and $100 \mathrm{mcg}$ for IM injections [16], so that the results could be easily compared between two nasal formulations. Baseline corrected data was considered as it gives a more accurate comparison of the performance of formulations.

In this study the bioavailability of Methylcobalamin Nasal Spray was found to be comparable to intramuscular injection; however upon dose normalization it was found to be $\sim 20 \%$. Bioavailability of commercial oral tablets of B12 has been reported to be low ( 2\%)[18]. As current nasal formulation of methylcobalamin is higher bioavailable it may lead to faster and reliable treatment of B12 deficiency as compared to oral treatment. Also in comparison to intramuscular injections it is convenient and does not require health care professionals for administration. Thus novel Methylcobalamin nasal spray can be used as an alternative to both oral and IM formulations for treatment of Vitamin B12 deficiency.

Available literature suggests that the relative bioavailability of the nasal formulations of cyanocobalamin and hydroxocobalamin (versus intramuscular) had been estimated to be $2-6.1 \%[16,19]$ and $2-5 \%$ [15], respectively. Thus this novel methylcobalamin nasal spray is demonstrating higher relative bioavailability than has been previously reported.

The $\mathrm{T}_{\max }$ of $12 \pm 3.6 \mathrm{~min}(0.20 \pm 0.06$ hours), observed in our study was quicker than that of $1.25 \pm 1.9$ hours with cyanocobalamin nasal spray, [16] and $35 \pm 13$ minutes observed with hydroxocobalamin nasal formulation [15].

Furthermore, the corresponding parameter $\mathrm{C}_{\max }$, reflecting the extent of absorption observed in our study was $19,383 \mathrm{pg} / \mathrm{mL}$ whereas for cyanocobalamin and hydroxocobalamin nasal formulations it was observed to be $758 \mathrm{pg} / \mathrm{mL}$ [16] and 1,716 pg/mL (derived from 1900 $\mathrm{pmol} / \mathrm{L}$ at $750 \mathrm{mcg}$ dose) [15] respectively. This would suggest that the absorption with the novel nasal formulation of methylcobalamin was higher than what has been documented for other nasal formulations of B12.

Cyanocobalamin intramuscular injections at $100 \mathrm{mcg}$ dose are used to treat $\mathrm{B} 12$ deficiency. The baseline corrected $\mathrm{C}_{\max }$ and $\mathrm{AUC}$ values observed in our study post IM injection of $100 \mathrm{mcg}$ methylcobalamin were comparable to those observed in the literature [20] with cyanocobalamin $100 \mathrm{mcg}$ IM injection. As the Methylcobalamin Nasal Spray (500 mcg dose) has similar bioavailability to that of a therapeutic dose for $(100 \mathrm{mcg}) \mathrm{IM}$ injections, it could offer an alternative for the treatment of vitamin B12 deficiency. Further, the baseline corrected mean concentration observed at 48 hours post dose with the $500 \mathrm{mcg}$ methylcobalamin nasal spray was $211.14 \pm 76.69 \mathrm{pg} /$ $\mathrm{mL}$ (corresponding to a rise in B12 level from 396.14 \pm 98.66 to 624.85 $\pm 145.50 \mathrm{pg} / \mathrm{mL}$ ). This suggested that an alternate-day dosing regimen could be adequate for correcting low B12 levels. The baseline corrected mean concentration observed after 5 days of dosing was $114.42 \pm 51.58 \mathrm{pg} / \mathrm{mL}$ (corresponding to a rise in B12 level from $396.14 \pm 98.66$ to $508.08 \pm 96.50 \mathrm{pg} / \mathrm{mL}$ ). This suggested that a weekly dose could be used to maintain the sufficient B12 levels post correction. These doses were subsequently evaluated in a clinical trial conducted on 103 vitamin B12 deficient patients (Clinical Trial Registry: CTRI/2015/06/005952).

The safety of methylcobalamin is well established and 500 mcg dose as IM/IV injection is approved in many parts of the world. This was also supported in our Bioavailability study, where single doses of Nasal Spray and IM injection were both found to be well tolerated.

\section{Conclusion}

Novel methylcobalamin $500 \mathrm{mcg} / 0.1 \mathrm{ml}$ nasal spray is painless, selfadministered, well-tolerated, and safe, provides a fast nasal absorption and has similar bioavailability to that of $100 \mathrm{mcg}$ IM injection. Thus it could offer an attractive alternative to current oral and injectable treatment options for Vitamin B12 deficiency.

\section{Declaration}

\section{Funding}

This research was funded by Troikaa Pharmaceuticals Limited.

Clinical trial registry, CTRI No. CTRI/2015/04/005687. 


\section{Conflicts of interest}

The authors declare no conflict of interest. The funders had no role in the design of the study; in the collection, analyses, or interpretation of data; in the writing of the manuscript, or in the decision to publish the results.

\section{Ethics Approval}

\section{Consent to participate}

Availability of data and material: Please contact Corresponding Author.

\section{Code availability}

Not applicable

\section{Consent for Publication and Author Contributions}

\section{Author contributions}

Conceptualization, methodology, study conduct, formal analysis, access to all data, verification of data and interpretation: Dave $\mathrm{H}$. All authors have read and agreed to the published version of the manuscript.

\section{Acknowledgment}

Investigational Product and support from Troikaa Pharmaceuticals Limited.

\section{References}

1. Langan RC, Goodbred AJ (2017) Vitamin B12 deficiency: Recognition and management. Am Fam Physician 96: 384-389.

2. Pawlak R, Lester SE, Babatunde T (2014) The prevalence of cobalamin deficiency among vegetarians assessed by serum vitamin B12: a review of literature. Eur J Clin Nutr 68: 541-548.

3. Green R, Allen LH, Bjørke-Monsen AL, Brito A, Jean-Louis G, et al. (2017) Vitamin B12 deficiency. Nat Rev Dis Primers 3: 17040.

4. Greenblatt DJ, Allen MD (1978) Intramuscular injection-site complications. JAMA 240: 542-544.

5. Sartorius G, Fennell C, Spasevska S, Turner L, Conway AJ, et al. (2010) Factors influencing time course of pain after depot oil intramuscular injection of testosterone undecanoate. Asian J Androl 12: 227-233.

6. Estourgie-van Burk GF, van der Kuy PH, de Meij TG, Benninga MA, Kneepkens CMF (2019) Intranasal treatment of Vitamin B12 deficiency in children. Eur J Pediatr 179: 349-352.
7. Van WC, Austin P, Naylor CD (2001) Vitamin B12 injections versus oral supplements. How much money could be saved by switching from injections to pills? Can Fam Physician 47: 79-86.

8. Chan CQH, Low LL, Lee KH (2016) Oral Vitamin B12 Replacement for the treatment of pernicious anemia. Front Med (Lausanne) 3: 38.

9. Kibirige $D$, Mwebaze $R$ (2013) Vitamin B12 deficiency among patients with diabetes mellitus: is routine screening and supplementation justified? J Diabetes Metab Disord 12: 17.

10. Stover PJ (2010) Vitamin B12 and older adults. Curr Opin Clin Nutr Metab Care 13: 24-27.

11. Türker $S$, Onur $E$, Ozer $Y$ (2004) Nasal route and drug delivery systems. Pharm World Sci 26: 137-142.

12. Ghori MU, Mahdi MH, Smith AM, Conway BR (2015) Nasal drug delivery systems: An overview. Am J Pharmacol Sci 3: 110-119.

13. Chhajed S, Sangale S, Barhate SD (2011) Advantageous nasal drug delivery system: a review. Int J Pharm Sci Res 2: 1322.

14. Rozgony NR, Fang C, Kuczmarski MF, Bob H (2010) Vitamin B(12) deficiency is linked with long-term use of proton pump inhibitors in institutionalized older adults: could a cyanocobalamin nasal spray be beneficial? J Nutr Elder 29: 87-99.

15. Van Asselt DZ, Merkus FW, Russel FG, Hoefnagels WH (1998) Nasal absorption of hydroxocobalamin in healthy elderly adults. Br J Clin Pharmacol 45: 83-86.

16. Nascobal Nasal Spray, Cyanocobalamin, USP $500 \mathrm{mcg} / \mathrm{spray}$ Pack Insert.

17. Kelly G (1997) The Coenzyme Forms of Vitamin B12: Toward an understanding of their Therapeutic Potential. Alt Med Rev 2: 459471.

18. Castelli MC, Wong DF, Friedman K, Riley MG (2011) Pharmacokinetics of oral cyanocobalamin formulated with sodium $\mathrm{N}$-[8-(2-hydroxybenzoyl)amino]caprylate (SNAC): an open-label, randomized, single-dose, parallel-group study in healthy male subjects. Clin Ther 33: 934-945.

19. Tillemans MPH, Donders EMVJ, Verweij SL, Van der Hoeven RTM, Kalisvaart KJ (2014) Effect of administration route on the pharmacokinetics of Cobalamin in elderly patients: a randomized controlled trial. Curr Ther Res Clin Exp 76: 21-25.

20. US Food and Drug Administration (2003-2004) Clinical Pharmacology and Biopharmaceutics Review(S). Nascobal Nasal spray (Cyanocobalamin Nasal Spray). 